\section{BMJ Open Respiratory Research}

\title{
Characteristics of pulmonary cryptococcosis in patients with rheumatoid arthritis
}

\author{
Chiaki Hosoda, ${ }^{1,2}$ Takashi Ishiguro, ${ }^{1}$ Ryuji Uozumi (D) , ${ }^{3}$ Miyuki Ueda, ${ }^{4}$ \\ Naomi Takata, ${ }^{4}$ Noboru Takayanagi ${ }^{1}$
}

To cite: Hosoda C, Ishiguro T, Uozumi R, et al. Characteristics of pulmonary cryptococcosis in patients with rheumatoid arthritis. BMJ Open Resp Res 2021;8:e000805. doi:10.1136/ bmjresp-2020-000805

- Additional supplemental material is published online only. To view, please visit the journal online (http://dx.doi. org/10.1136/bmjresp-2020000805).

Received 30 October 2020 Accepted 5 July 2021

Check for updates

(c) Author(s) (or their employer(s)) 2021. Re-use permitted under CC BY-NC. No commercial re-use. See rights and permissions. Published by BMJ.

${ }^{1}$ Department of Respiratory

Medicine, Saitama

Cardiovascular and

Respiratory Center, Saitama, Japan

${ }^{2}$ Division of Respiratory Diseases, Department of Internal Medicine, The Jikei University School of Medicine, Tokyo, Japan

${ }^{3}$ Department of Biomedical Statistics and Bioinformatics, Kyoto University Graduate School of Medicine, Kyoto, Japan

${ }^{4}$ Department of Radiology, Saitama Cardiovascular and Respiratory Center, Saitama, Japan

Correspondence to Dr Chiaki Hosoda; ms03hosoda@jikei.ac.jp

\section{ABSTRACT}

Background and objective A high frequency of infections complicating rheumatoid arthritis (RA) has been reported due to the immunomodulatory effect of RA or to agents with immunosuppressive effects used in its treatment. We aimed to assess clinical and radiological characteristics of pulmonary cryptococcosis in patients with and without RA. Methods We retrospectively reviewed the medical records of 52 patients with pulmonary cryptococcosis and divided them into two groups, those with RA and without RA, and compared clinical characteristics and radiological findings between them.

Results Eleven (21.2\%) of the 52 patients had RA. Median follow-up periods were 51.2 (range: 1.1-258.7) months for patients with RA and 19.1 (range: 0.63-246.9) months for patients without RA. Among the patients with RA, $81.8 \%$ were women, with a mean age of 68.1 years. Female sex and respiratory comorbidities were significantly more frequent in patients with RA than in patients without RA. Frequencies of concomitant cryptococcal meningitis and respiratory failure were not different between the groups. There were no significant differences in frequency of any radiological findings, locations and number between the two groups. Among patients with RA, all but one responded well to antifungal treatment. During the antifungal treatment course, one $(9.1 \%)$ patient with RA died of cryptococcosis. Despite continuing antirheumatic drugs, no patients had recurrence of pulmonary cryptococcosis during follow-up.

Conclusion Other than some differences in background, there were no clinical, radiological or prognostic differences between the patients with and without RA with pulmonary cryptococcosis. The administration of antirheumatic therapy had no negative effect on the clinical course of antifungal treatment.

\section{INTRODUCTION}

Rheumatoid arthritis (RA) is an autoimmune disease resulting in chronic systemic inflammation that affects many tissues and organs. A high frequency of infections complicating RA has been reported due to the immunomodulatory effect of RA or agents with immunosuppressive effects used in its treatment. ${ }^{1}$ Cryptococcosis is a fungal infection caused by encapsulated yeast in the genus Cryptococcosis that
Key messages

\section{What is the key question?}

- What are the clinical and radiologic differences between pulmonary cryptococcosis in patients with $\mathrm{RA}$ and without RA and the clinical courses of both pulmonary cryptococcosis and antirheumatic drug therapy after the diagnosis of cryptococcosis?

\section{What is the bottom line?}

- Minimal differences were found in the clinical and radiologic findings of the patients with RA, and the administration of antirheumatic therapy during and after treatment for cryptococcosis did not negatively affect the clinical course of antifungal treatment.

\section{Why read on?}

- Although the small sample size makes it difficult to draw any firm conclusions, antirheumatic drugs were continued safely during and after antifungal treatment for cryptococcosis, and this study offers preliminary data for further prospective studies.

enter the human body through inhalation. ${ }^{23}$ Approximately $20 \%$ of patients diagnosed as having cryptococcosis have no apparent risk factors or underlying disease. ${ }^{4}$ Cryptococcosis is now observed more often in patients with RA who are immunocompromised by treatment. Although pulmonary cryptococcosis may be one of the most important infectious complications of patients with RA, there are few case reports and studies of pulmonary cryptococcosis in these patients. ${ }^{5-13}$ The clinical and radiological characteristics of pulmonary cryptococcosis in patients with RA have not been fully elucidated. Moreover, whether immunosuppressant drugs can be continued and which antirheumatic drugs can be used safely and efficiently when cryptococcosis develops remain unclear.

This study aimed to clarify the clinical characteristics, radiologic findings and prognosis of pulmonary cryptococcosis in patients with RA, and the safety of 
antirheumatic drugs in patients with RA after a diagnosis of cryptococcosis.

\section{METHODS}

Patients

We identified 52 patients diagnosed as having pulmonary cryptococcosis at Saitama Cardiovascular and Respiratory Center between January 1997 and August 2018. Overall, 11 patients with RA and 41 patients without RA were included in this study.

\section{Study design}

We retrospectively reviewed the medical records of these 52 patients. The diagnosis of pulmonary cryptococcosis was defined as either (1) identification of the cryptococcal microorganism by morphological identification or microbial culture in lung biopsy specimen, bronchial washing fluid or bronchoalveolar lavage fluid; or (2) positive results of serum cryptococcal antigen, with clinical and radiological evidence of active pulmonary infection. All cryptococcal antigen titres were measured with a commercially available latex agglutination test (Eiken, Tokyo, Japan). Cryptococcal meningitis was determined either by symptoms of suspected meningitis (eg, headache and impaired consciousness) and involvement of the central nervous system with Cryptococcus in histology or culture of brain or cerebral spinal fluid.

Baseline clinical characteristics were obtained within 1 month of the initial diagnosis of cryptococcosis. The clinical course of the patients who died during antifungal treatment and received tumour necrosis factor (TNF) blockage therapy before developing pulmonary cryptococcosis were reviewed.

RA was diagnosed by rheumatologists certified by the American College of Rheumatology/European League Against Rheumatism Criteria. ${ }^{14}$ The treatment strategies with antirheumatic drugs were planned by the rheumatologists who introduced each patient to our hospital. The study protocol was approved by the Ethics Committee of Saitama Cardiovascular and Respiratory Center (Approval no. 2018046) and was conducted in compliance with the Declaration of Helsinki.

\section{Interpretation of chest CT scans}

The CT findings of 33 of the 52 patients with pulmonary cryptococcosis were analysed. Eight patients had RA and 25 did not. CT films were not available for the remaining 19 patients. All images were independently reviewed by two experienced radiologists (MU and NT) blinded to the clinical information. The final reading was reached by consensus.

Findings of the chest CT scans were assessed for (1) the presence and distribution of parenchymal lesions, including nodules, masses and consolidation; (2) characteristics of the nodules and masses; and (3) related thoracic abnormalities such as pleural effusion and lymphadenopathy according to previous reports. ${ }^{12} 1516$ Based on the predominant parenchymal findings on CT, the morphological characteristics were classified as solitary nodule/mass, multiple nodules/masses and consolidation. Multiple nodules/masses were further subdivided into distribution in a single lobe and distribution in multiple lobes.

\section{Statistical analysis}

Categorical variables are reported as numbers and percentages and were compared with Fisher's exact test. Continuous variables are presented as mean $\pm \mathrm{SD}$ and were compared with the Student t-test. A $p$ value $<0.05$ was considered significant. Interobserver reliability of the radiological findings was evaluated using the kappa coefficient $(\kappa)$ and was defined as poor $(\kappa<0.00)$, slight $(0.00$ $0.20)$, fair $(0.21-0.40)$, moderate $(0.41-0.60)$, substantial (0.61-0.80) and almost perfect (0.81-1.00). All statistical analyses were performed with SAS V.9.4 (SAS Institute, Inc, Cary, North Carolina, USA).

\section{Patient and public involvement}

Patients were not involved in the planning, design, data collection or the conduct of this study. The study results will be disseminated through scientific journals.

\section{RESULTS}

\section{Clinical and laboratory findings}

Eleven (21.2\%) of the 52 patients had RA, and 15 (28.8\%) had underlying immunosuppressive diseases other than RA. All patients were HIV negative. All patients (98.1\%) had Cryptococcus neoformans except for one (1.9\%) with $C$. gattii. Clinical characteristics and laboratory findings of the 11 patients with RA with pulmonary cryptococcosis are listed in table 1 .

Among the patients with RA, $9(81.8 \%)$ were women, with a mean age of $68.1 \pm 8.8$ years. Underlying respiratory diseases were identified in $7(63.6 \%)$ patients. Cerebrospinal fluid obtained via lumbar puncture was examined in $3(27.3 \%)$ patients, and $1(0.9 \%)$ patient was positive for Grocott's stain and culture.

Four patients $(36.4 \%)$ were asymptomatic and were detected incidentally by mass screening examination or routine X-ray check. Fever was the most common presenting symptom followed by cough. However, of the four patients with RA with fever, one had meningitis and another had concomitant methotrexate (MTX) pneumonitis.

Compared with the patients without RA, the mean age of the patients with RA was significantly older, and the ratio of females, the frequency of underlying respiratory disease, and that of fever as an initial symptom were significantly higher in the patients with RA (online supplemental table 1 ). 
Table 1 Clinical characteristics and laboratory findings of the 11 patients with rheumatoid arthritis

\begin{tabular}{lc}
\hline & Value \\
\hline Female & $9(81.8 \%)$ \\
\hline Age, mean \pm SD (years) & $68.1 \pm 8.8$ \\
\hline Smoker & $2(18.2 \%)$ \\
\hline Cryptococcal meningitis & $1(9.1 \%)$ \\
\hline Respiratory failure & $2(18.2 \%)$ \\
\hline Comorbidity & \\
\hline Respiratory disease & $7(63.6 \%)$ \\
\hline Interstitial pneumonia & $1(9.1 \%)$ \\
\hline Pulmonary emphysema & 0 \\
\hline Previous pulmonary tuberculosis & $1(9.1 \%)$ \\
\hline Bronchiolitis & $2(18.2 \%)$ \\
\hline Non-tuberculosis mycobacteria & $2(18.2 \%)$ \\
\hline Others & $1(9.1 \%)$ \\
\hline Non-respiratory disease & $1(9.1 \%)$ \\
\hline Diabetes mellitus & 0 \\
\hline Advanced solid tumour & 0 \\
\hline Chronic kidney disease & 0 \\
\hline Chronic liver disease & $1(9.1 \%)$ \\
\hline Immunosuppressive therapy or & $10(90.9 \%)$ \\
\hline chemotherapy & $2(18.2 \%)$ \\
\hline Others & $10(90.9 \%)$ \\
\hline Symptoms & \\
\hline Asymptomatic & $5(45.5 \%)$ \\
\hline Fever & $4(36.4 \%)$ \\
\hline Cough & $4(36.4 \%)$ \\
\hline General fatigue & $1(9.1 \%)$ \\
\hline Impaired consciousness & 0 \\
\hline Dyspnoea & $1(9.1 \%)$ \\
\hline antigen & $143855 \pm 4292$ \\
\hline Nymeutre cell count (mm $\left.{ }^{3}\right)$ & $5616 \pm 2508$ \\
\hline
\end{tabular}

The white blood cell (WBC) counts and $\mathrm{C}$ reactive protein (CRP) levels were significantly higher in the patients with RA .

\section{Radiologic features}

The pulmonary abnormalities seen on initial CT scans of the patients with RA are summarised in table 2 . The most common CT finding among the patients $(50 \%)$ with RA was consolidation. There were no significant differences in the locations, number and frequency of any CT findings between the patients with and without RA (online supplemental table 2).
Table 2 High-resolution CT findings in eight patients with rheumatoid arthritis

\begin{tabular}{|c|c|}
\hline & Value \\
\hline Solitary nodule or mass & $3(37.5 \%)$ \\
\hline Multiple nodules in single lobe limited & $1(12.5 \%)$ \\
\hline Multiple nodules in multiple lobes & 0 \\
\hline Consolidations & $4(50.0 \%)$ \\
\hline Nodules and masses & $7(87.5 \%)$ \\
\hline $\begin{array}{l}\text { Multiple nodules/masses in the same lung } \\
\text { lobe }\end{array}$ & $4(50.0 \%)$ \\
\hline \multicolumn{2}{|l|}{ Number of involved lobes } \\
\hline 1 & $5(62.5 \%)$ \\
\hline 2 & 0 \\
\hline 3 & 0 \\
\hline 4 & $2(25.0 \%)$ \\
\hline 5 & $1(12.5 \%)$ \\
\hline \multicolumn{2}{|l|}{ Size $(\mathrm{mm})$} \\
\hline $1-30$ & 7 (87.5\%) \\
\hline$\geq 31$ & 0 \\
\hline \multicolumn{2}{|l|}{ Number } \\
\hline 1 & $3(37.5 \%)$ \\
\hline $2-4$ & $2(25.0 \%)$ \\
\hline $5-9$ & 0 \\
\hline$\geq 10$ & $2(25.0 \%)$ \\
\hline \multicolumn{2}{|l|}{ Border } \\
\hline Well-defined & $7(87.5 \%)$ \\
\hline III-defined & 0 \\
\hline Convergence of bronchi and vessel & 0 \\
\hline Pleural indentation & 0 \\
\hline Consolidation & $4(50.0 \%)$ \\
\hline \multicolumn{2}{|l|}{ Number of involved lobes } \\
\hline 1 & $1(12.5 \%)$ \\
\hline 2 & 0 \\
\hline 3 & $2(25.0 \%)$ \\
\hline 4 & 0 \\
\hline 5 & $1(12.5 \%)$ \\
\hline \multicolumn{2}{|l|}{ Internal characteristics } \\
\hline Air bronchogram & $5(62.5 \%)$ \\
\hline Cavitation & $4(50.0 \%)$ \\
\hline Calcification & 0 \\
\hline Halo sign & $2(25.0 \%)$ \\
\hline Satellite lesion & $5(62.5 \%)$ \\
\hline Pleural effusion & 0 \\
\hline Lymphadenopathy & $1(12.5 \%)$ \\
\hline
\end{tabular}

Antifungal treatment and in-hospital outcome

Treatment for pulmonary cryptococcosis in the patients with RA is summarised in table 3 . The mainstay of initial 


\begin{tabular}{lc}
\hline $\begin{array}{l}\text { Table } 3 \quad \text { Antifungal treatment in the } 11 \text { patients with } \\
\text { rheumatoid arthritis }\end{array}$ & Value \\
\hline & 0 \\
\hline No treatment & 0 \\
Surgery without antifungal drugs & $1(9.1 \%)$ \\
Surgery with antifungal drugs & $11(100.0 \%)$ \\
Antifungal drugs & $8(72.7 \%)$ \\
\hline Azoles alone & 8 \\
Fluconazole & 0 \\
Voriconazole & 0 \\
Itraconazole & $3(27.3 \%)$ \\
Azoles and 5-fluorocytosine & 0 \\
\hline Amphotericin B and 5-fluorocytosine & \\
\hline
\end{tabular}

treatment in both groups was azoles. All patients with RA received antifungal treatment. Among the patients with RA, $8(72.7 \%)$ were initially treated with fluconazole (FLCZ) and 3 (27.3\%) with FLCZ and 5-fluorocytosine (FC). The median duration (range) of treatment was 6 (3-61) months. Two patients with RA discontinued FLCZ at 4.5 and 3 months due to alopecia and oedema, respectively.

One $(9.1 \%)$ patient with RA died during hospitalisation, whereas no patients without RA died. The fatality was a 78-year-old man with an 8-year history of RA receiving MTX of $8 \mathrm{mg}$ weekly and prednisolone of 2.5 $\mathrm{mg}$ daily. The initial symptoms were fever $\left(38^{\circ} \mathrm{C}\right)$ for 1 month with respiratory failure. Chest CT findings showed consolidations in four lobes of the lung. Cerebrospinal fluid cultures were sterile and negative for Grocott's stain. The patient did not respond to antifungal treatment (FLCZ and VRCZ) and died of respiratory failure due to progression of cryptococcosis during hospitalisation.

\section{Clinical course after discharge}

Among the 52 patients, 44 were followed for $>6$ months (median of 51.2 (1.1-258.7) months for patients with RA and 19.1 (0.63-246.9) months for patients without RA). Twelve patients showed disappearance of radiological abnormalities, and the remaining 32 patients showed reduction of radiological abnormalities without regrowth. One patient without RA with cryptococcal meningitis died of aspiration pneumonia after discharge. No patients experienced recurrence of pulmonary cryptococcosis during follow-up.

\section{Clinical courses of patients and antirheumatic treatment after} pulmonary cryptococcosis diagnosis and during follow-up

The clinical courses of antifungal and antirheumatic treatment in the patients with RA are described in table 4. Antirheumatic drugs were discontinued at the diagnosis of cryptococcosis in six patients. In patient \#1, MTX pneumonitis was diagnosed simultaneously based on established criteria. ${ }^{17}$ MTX was discontinued, and pulse methylprednisolone followed by oral prednisolone at $60 \mathrm{mg}$ daily was initiated. The postdiagnosis management of cryptococcosis in the other five patients included discontinuation of MTX (patients \#5, \#11), MTX and infliximab (patient \#6), mizoribine (patient \#7), and tacrolimus (patient \#10). Symptoms indicating deterioration of RA recurred in all five patients within 1 month from discontinuation. All patients other than the patient who died of cryptococcosis (atient \#11) were readministered the same drugs.

Only one patient received anti-TNF therapy before developing pulmonary cryptococcosis (table 4, patient \#6). TNF blockade was begun with infliximab at 6 months before developing cryptococcosis. Chest CT performed prior to infliximab administration revealed a few faint nodules (figure 1A). Six months after the initiation of infliximab, the patient complained of fever and cough, and CT scans revealed consolidation in the right lower lobe (figure 1B). Pulmonary cryptococcosis was diagnosed by bronchial washing fluid culture, which showed C. neoformans. Infliximab was then stopped but was restarted 2 months after the initiation of FLCZ because of deterioration of RA symptoms. The pulmonary cryptococcosis improved and has not relapsed for 8 years.

\section{DISCUSSION}

We assessed the clinical characteristics, radiologic findings and outcome of pulmonary cryptococcosis in patients with RA. Minor clinical differences were observed between the patients with and without RA, although no significant differences were observed in the frequencies of meningitis and respiratory failure and radiological findings. All but one patient with RA had a favourable outcome. Administration of antirheumatic drugs during and after treatment for cryptococcosis had no negative effects on the clinical course of antifungal treatment in terms of rate of treatment success and recurrence.

Among the patients with pulmonary cryptococcosis, $21.2 \%$ had RA. The prevalence of RA in Japan was estimated at between $0.6 \%$ and $1.0 \% .{ }^{18}$ However, the proportion of patients with RA with pulmonary cryptococcosis in this study was much higher than the prevalence of RA in the general population. C. neoformans is a facultative intracellular pathogen, and one important protective function against infection is cellular immunity. ${ }^{19}$ Host defence against $C$. neoformans infection is mediated by $\mathrm{T}$ helper type 1 cellular immunity, which is triggered by host cell recognition of the pathogen-associated molecular pattern recognition receptors. ${ }^{20}$ Rheumatoid inflammation is suggested to be mediated by activated proinflammatory $\mathrm{T}$ helper type 1 cells, and thus, the impaired host as defined by patients with RA may contribute to the high prevalence of pulmonary cryptococcosis. We also found that patients with RA with pulmonary cryptococcosis were predominantly female and older than the patients without RA. Many studies of pulmonary cryptococcosis 


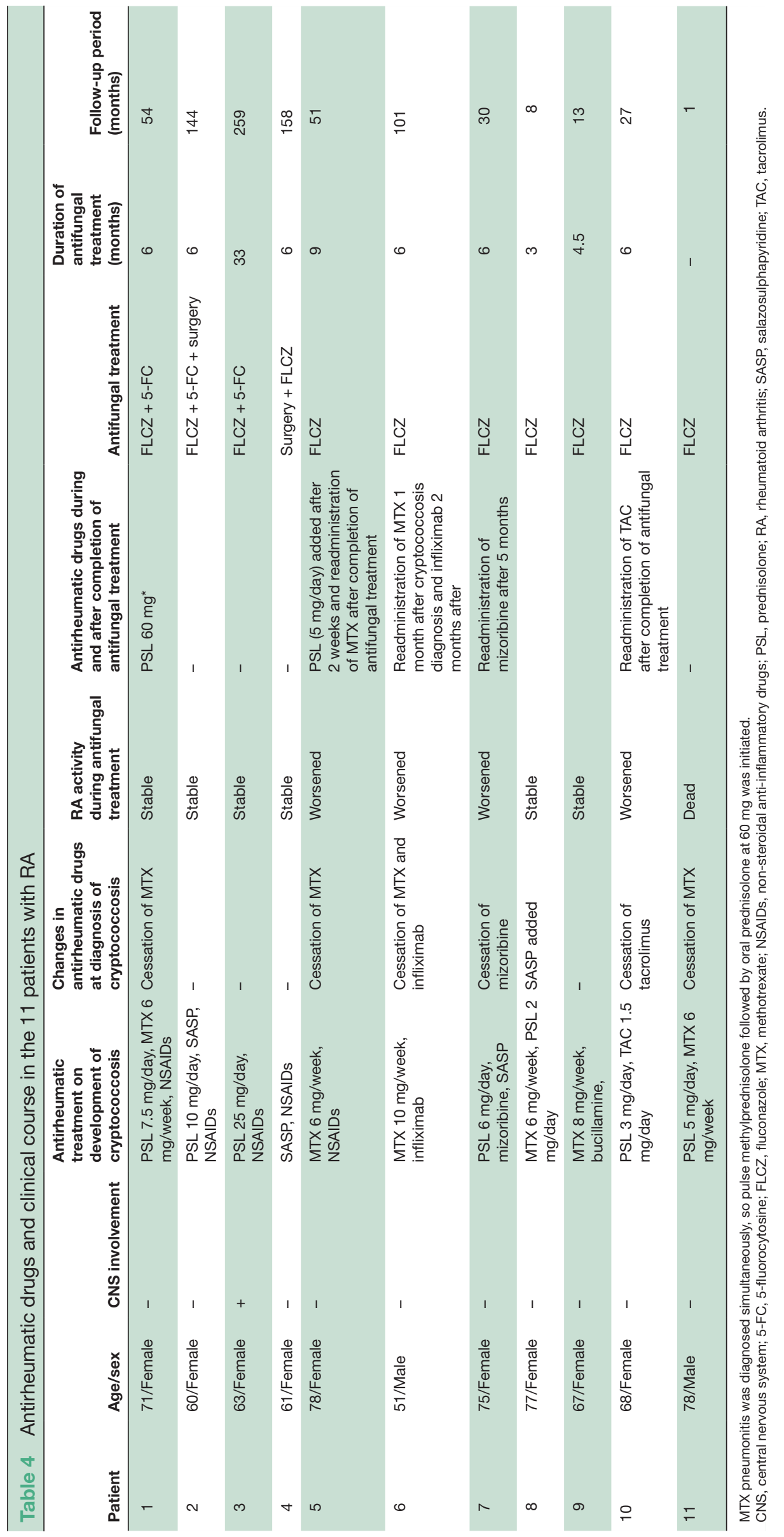




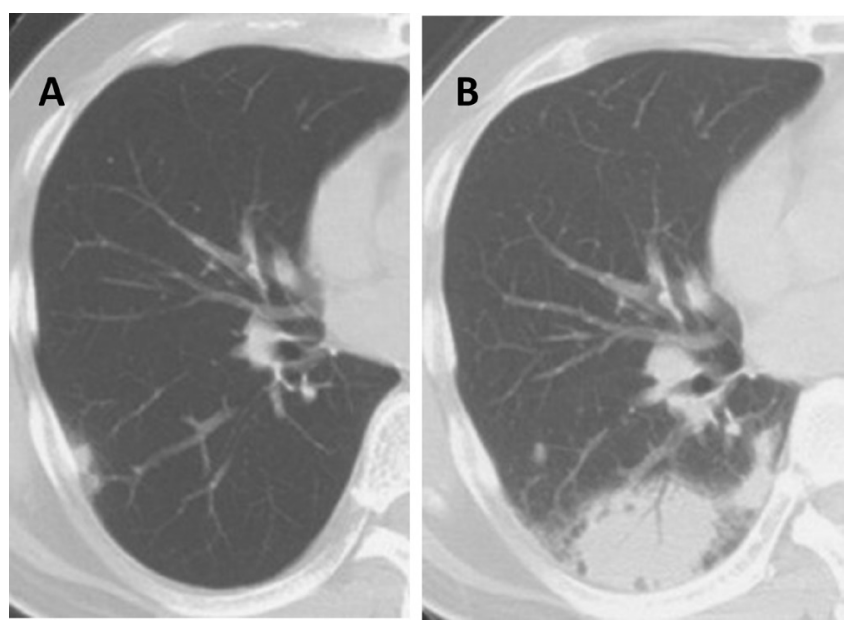

Figure 1 Chest CT findings in the patient with rheumatoid arthritis treated with infliximab. (A) CT image prior to initiation of infliximab shows a few faint nodules (arrow). (B) Six months after the initiation of infliximab, a CT image reveals consolidation in the right lower lobe.

have shown male predominance or almost equal numbers of males and females. ${ }^{21}{ }^{22}$ Our findings may reflect the higher age and female predominance observed in the RA population overall.

Fever was the most common presenting symptom, and the WBC counts and CRP levels were significantly higher in the patients with RA. Fever was less frequent (0\%-25\%) among patients with pulmonary cryptococcosis without HIV infection. ${ }^{21}{ }^{22}$ Yanagawa et al reported that hyper-reaction to organisms was seen in pulmonary cryptococcosis with patients with RA pathologically and was different from the reaction protecting against infection. ${ }^{12}$ This reaction was also reported in patients with RA with Pneumocystis pneumonia. ${ }^{23}$ Moreover, because 7 of the 11 patients with RA were taking steroid, WBCs might have been elevated due to this drug. Also, CRP might be one of the inflammatory markers of RA itself.

Chest CT imaging showed consolidation to be the most common finding, although no significant differences in any findings were observed between the patients with and without RA. Among immunocompetent patients and individuals without AIDS, the most common CT feature was the presence of multiple peripherally distributed pulmonary nodules or masses with predominantly lower lobe involvement, ${ }^{15} 21$ 24-28 although the number of nodules or masses in previous reports has varied. Cavitation in nodules or masses and pleural effusion have been previously described as radiographic features limited to immunosuppressed patients. ${ }^{29}{ }^{30}$ However, consolidation and ground-glass attenuation were the main radiologic features in patients with RA, with no differences in the frequency of cavitary lesions and pleural effusion, which were compatible with our results. Among seven reported patients who received anti-TNF biological therapy, including our patient, three $(42.9 \%)$ showed a consolidation pattern, one $(14.3 \%)$ had multiple nodules and two $(28.6 \%)$ showed a solidary nodule/mass pattern. RA itself and treatment for RA may not necessarily affect the variation in radiologic findings.

Among our patients with RA, 72.7\% received FLCZ, and the remainder received FLCZ and 5-FC. All but one patient improved and did not have recurrence of cryptococcosis. The guidelines for cryptococcosis management in Japan recommend FLCZ for 3-6 months in patients without central nervous system involvement. ${ }^{31}$ Conversely, the Infectious Diseases Society of America guideline for pulmonary cryptococcosis in non-immunosuppressed patients without HIV states that for mild-to-moderate symptoms, FLCZ (400 mg/day orally) should be administered for 6-12 months. ${ }^{32}$ All of our patients with RA without meningitis received FLCZ \pm 5 FC for $<9$ months, whereas one patient with meningitis received it for 61 months. No patients, including two in whom FLCZ was withdrawn at 3 and 4.5 months, respectively, due to side effects, experienced disease recurrence. These findings suggested that the duration of treatments as recommended in the Japanese guidelines ${ }^{31}$ may also be appropriate in patients with RA. Further studies are needed to determine the optimal duration of treatment for cryptococcosis in patients with RA .

Cryptococcosis is a severe fungal infection with high mortality rate among organ transplant recipients and patients with AIDS. ${ }^{33}$ In contrast, several studies have shown favourable outcomes of pulmonary cryptococcosis among patients without HIV. ${ }^{15} 16$ Only two $(3.8 \%)$ of our patients died during the course of antifungal treatment, including one patient each in the RA and non-RA groups. The patient with RA died due to pulmonary cryptococcosis, and the patient without RA complicated with meningitis developed aspiration pneumonia and deteriorated to death. Pulmonary cryptococcosis itself could be a fatal infectious complication in patients with RA. Due to the small sample number in the present study, further investigation is required into differences in mortality between patients with and without RA.

Several studies have shown that the occurrence of opportunistic infection was significantly higher in patients with RA who received TNF blockage therapy ${ }^{1334}$ As TNF production is critical in the immune response against cryptococcal infection, ${ }^{35}$ depletion of TNF by treatment with TNF blockage therapy might facilitate the development of cryptococcosis. Seven cases, including our case, have been reported of pulmonary cryptococcosis complicated with RA in patients receiving TNF blockage therapy. ${ }^{5-10}$ Among them, cryptococcosis occurred within 6 months of initiating biologics in all cases, suggesting that infectious complications can occur early. All patients responded well to antifungal treatment. It is important that physicians include pulmonary cryptococcosis in the differential diagnosis when they encounter patients with RA with compatible radiological findings or clinical findings with pulmonary cryptococcosis in an early stage after initiation of TNF blockage therapy.

Long-term administration of immunosuppressant drugs carries the risk of several complications and can result in subsequent or prolonged infection. It is not clear whether 
immunosuppressant drugs can be continued when developing cryptococcal infections and whether antirheumatic drugs can be used safely and efficiently in such situations. The present study showed that rheumatologists hesitated to continue antirheumatic drugs that were administered prior to the cryptococcosis diagnosis during antifungal treatment. RA activities in our patients worsened with discontinuation of the antirheumatic drugs. Their readministration had no negative effects on the clinical course of antifungal treatment and did not increase the risk of relapse of cryptococcosis. From the present results, we believe that it is reasonable to assume that antirheumatic drugs could be continued during antifungal treatment for cryptococcosis. Further studies are needed to establish adequate strategies for treating RA when developing cryptococcosis.

This study has several limitations. First, it is a singlecentre retrospective study, which reduces the level of confidence, and the results may not be applicable in other settings. Second, the small sample size makes it difficult to draw any firm conclusions. It may be premature to draw a conclusion that the administration of antirheumatic drugs during and after the development of pulmonary cryptococcosis in patients with RA is safe. The accumulation of more cases and the conduction of further investigations are needed in the future. Third, we did not investigate disease severity of RA because RA severity at the onset of cryptococcosis was noted in only a few of the patients' medical records.

In conclusion, a high prevalence of patients with RA with pulmonary cryptococcosis was noted. Only minimal differences were found in the clinical and radiological findings of the patients with and without RA. The administration of antirheumatic therapy during and after treatment for cryptococcosis did not negatively affect the clinical course of antifungal treatment. Further studies are needed to clarify the safety of antirheumatic drugs in patients with RA after the diagnosis of cryptococcosis and the optimal treatment approaches for cryptococcosis in the patients with RA.

Contributors All authors had full access to all the data in the study and had final responsibility for the decision to publish.

Funding The authors have not declared a specific grant for this research from any funding agency in the public, commercial or not-for-profit sectors.

Competing interests None declared.

Patient consent for publication Not required.

Provenance and peer review Not commissioned; externally peer reviewed.

Data availability statement No data are available.

Supplemental material This content has been supplied by the author(s). It has not been vetted by BMJ Publishing Group Limited (BMJ) and may not have been peer-reviewed. Any opinions or recommendations discussed are solely those of the author(s) and are not endorsed by BMJ. BMJ disclaims all liability and responsibility arising from any reliance placed on the content. Where the content includes any translated material, BMJ does not warrant the accuracy and reliability of the translations (including but not limited to local regulations, clinical guidelines, terminology, drug names and drug dosages), and is not responsible for any error and/or omissions arising from translation and adaptation or otherwise.

Open access This is an open access article distributed in accordance with the Creative Commons Attribution Non Commercial (CC BY-NC 4.0) license, which permits others to distribute, remix, adapt, build upon this work non-commercially, and license their derivative works on different terms, provided the original work is properly cited, appropriate credit is given, any changes made indicated, and the use is non-commercial. See: http://creativecommons.org/licenses/by-nc/4.0/.

ORCID iD

Ryuji Uozumi http://orcid.org/0000-0002-9546-9869

\section{REFERENCES}

1 Doran MF, Crowson CS, Pond GR, et al. Frequency of infection in patients with rheumatoid arthritis compared with controls: a population-based study. Arthritis Rheum 2002;46:2287-93.

2 Limper AH, Knox KS, Sarosi GA. American thoracic Society fungal Working Group. An official American thoracic Society statement: treatment of fungal infections in adult pulmonary and critical care patients. Am J Respir Crit Care Med 2011;183:96-128.

3 Kwon-Chung KJ, Varma A. Do major species concepts support one two or more species within. Cryptococcus neoformans? FEMS Yeast Res 2006;6:574-87.

4 La Hoz RM, Pappas PG. Cryptococcal infections: changing epidemiology and implications for therapy. Drugs 2013;73:495-504.

5 True DG, Penmetcha M, Peckham SJ. Disseminated cryptococcal infection in rheumatoid arthritis treated with methotrexate and infliximab. J Rheumatol 2002;29:1561-3.

6 Cadena J, Thompson GR, Ho TT, et al. Immune reconstitution inflammatory syndrome after cessation of the tumor necrosis factor alpha blocker adalimumab in cryptococcal pneumonia. Diagn Microbiol Infect Dis 2009;64:327-30.

7 Shrestha RK, Stoller JK, Honari G, et al. Pneumonia due to Cryptococcus neoformans in a patient receiving infliximab: possible zoonotic transmission from a pet cockatiel. Respir Care 2004;49:606-8.

8 Hage CA, Wood KL, Winer-Muram HT, et al. Pulmonary cryptococcosis after initiation of anti-tumor necrosis factor-alpha therapy. Chest 2003;124:2395-7.

9 Kozic H, Riggs K, Ringpfeil F, et al. Disseminated Cryptococcus neoformans after treatment with infliximab for rheumatoid arthritis. $J$ Am Acad Dermatol 2008;58:S95-6.

10 Iwata T, Nagano T, Tomita M, et al. Adalimumab-associated pulmonary cryptococcosis. Ann Thorac Cardiovasc Surg 2011;17:390-3.

11 Morita S, Shirai T, Asada K, et al. Pulmonary cryptococcosis presenting with a large cavity. Respirol Case Rep 2014;2:61-3.

12 Yanagawa N, Sakai F, Takemura T, et al. Pulmonary cryptococcosis in rheumatoid arthritis $(\mathrm{rA})$ patients: comparison of imaging characteristics among RA, acquired immunodeficiency syndrome, and immunocompetent patients. Eur J Radiol 2013;82:2035-42.

13 Liao T-L, Chen Y-M, Chen D-Y. Risk factors for cryptococcal infection among patients with rheumatoid arthritis receiving different immunosuppressive medications. Clin Microbiol Infect 2016;22:815. e1-815.e3.

14 Aletaha D, Neogi T, Silman AJ. Rheumatoid arthritis classification criteria: an American College of Rheumatology/European League against rheumatism collaborative initiative. Ann Rheum Dis 2010;2010:1580-8.

15 Chang W-C, Tzao C, Hsu H-H, et al. Pulmonary cryptococcosis: comparison of clinical and radiographic characteristics in immunocompetent and immunocompromised patients. Chest 2006;129:333-40.

16 Kohno S, Kakeya H, Izumikawa K, et al. Clinical features of pulmonary cryptococcosis in non-HIV patients in Japan. $J$ Infect Chemother 2015;21:23-30.

17 Camus P, Fanton A, Bonniaud P, et al. Interstitial lung disease induced by drugs and radiation. Respiration 2004;71:301-26.

18 Yamanaka H, Sugiyama N, Inoue E, et al. Estimates of the prevalence of and current treatment practices for rheumatoid arthritis in Japan using reimbursement data from health insurance societies and the IORRA cohort (I). Mod Rheumatol 2014;24:33-40.

19 Feldmesser M, Tucker S, Casadevall A. Intracellular parasitism of macrophages by Cryptococcus neoformans. Trends Microbiol 2001;9:273-8.

20 Ishii K, Kawakami K. [Pattern recognition and host defense response to Cryptococcus neoformans]. Med Mycol J 2012;53:247-54.

21 Kishi K, Homma S, Kurosaki A, et al. Clinical features and highresolution CT findings of pulmonary cryptococcosis in non-AIDS patients. Respir Med 2006;100:807-12.

22 Yu J-Q, Tang K-J, Xu B-L, JQ Y, BL X, et al. Pulmonary cryptococcosis in non-AIDS patients. Braz $J$ Infect Dis 2012;16:531-9. 
23 Tokuda H, Sakai F, Yamada H, et al. Clinical and radiological features of Pneumocystis pneumonia in patients with rheumatoid arthritis, in comparison with methotrexate pneumonitis and Pneumocystis pneumonia in acquired immunodeficiency syndrome: a multicenter study. Intern Med 2008;47:915-23.

24 Murayama S, Sakai S, Soeda H, et al. Pulmonary cryptococcosis in immunocompetent patients: HRCT characteristics. Clin Imaging 2004;28:191-5.

25 Lindell RM, Hartman TE, Nadrous HF, et al. Pulmonary cryptococcosis: CT findings in immunocompetent patients. Radiology 2005;236:326-31.

26 Fox DL, Müller NL. Pulmonary cryptococcosis in immunocompetent patients: CT findings in 12 patients. AJR Am J Roentgenol 2005;185:622-6.

27 Choe YH, Moon H, Park SJ, et al. Pulmonary cryptococcosis in asymptomatic immunocompetent hosts. Scand J Infect Dis 2009;41:602-7.

28 Wu B, Liu H, Huang J, et al. Pulmonary cryptococcosis in non-AIDS patients. Clin Invest Med 2009;32:70-7.
29 Khoury MB, Godwin JD, Ravin CE, et al. Thoracic cryptococcosis: immunologic competence and radiologic appearance. AJR Am J Roentgenol 1984;142:893-6.

30 Zinck SE, Leung AN, Frost M, et al. Pulmonary cryptococcosis: CT and pathologic findings. J Comput Assist Tomogr 2002;26:330-4.

31 Clinical practice guidelines for diagnosis and treatment of cryptococcosis. Tokyo, Japan: The Japanese Society for Medical Mycology, 2019.

32 Perfect JR, Dismukes WE, Dromer F, et al. Clinical practice guidelines for the management of cryptococcal disease: 2010 update by the infectious diseases Society of America. Clin Infect Dis 2010;50:291-322.

33 Husain S, Wagener MM, Singh N. Cryptococcus neoformans infection in organ transplant recipients: variables influencing clinical characteristics and outcome. Emerg Infect Dis 2001;7:375-81.

34 Kourbeti IS, Ziakas PD, Mylonakis E. Biologic therapies in rheumatoid arthritis and the risk of opportunistic infections: a metaanalysis. Clin Infect Dis 2014;58:1649-57.

35 Fa Z, Xu J, Yi J. TNF- $\alpha$-producing Cryptococcus neoformans exerts protective effects on host defenses in murine pulmonary cryptococcosis. Front Immunol 2019:10. 